ISSN: 0212-0267

DOI: http://dx.doi.org/IO.I420I/hedu2OI736277300

\title{
LA INCORPORACIÓN DE LAS MUJERES A LA SEGUNDA ENSEÑANZA PÚBLICA EN EXTREMADURA (1900-1936)
}

\section{The incorporation of women into the Secondary Public Education in Extremadura (1900-1936)}

Cristina Redondo Castro, Isabel Grana Gil y Carmen Sanchidrí́n Blanco

Teoría e Historia de la Educación. Facultad de Ciencias de la Educación.

Universidad de Málaga. Campus de Teatinos, 2907I Málaga (España)

Correo-e: cristinaredondo@uma.es; imgrana@uma.es; sanchidrian@uma.es

Recepción: 30 de marzo de 20I6. Envío a informantes: 29 de julio de 2016.

Aceptación definitiva: I4 de marzo de 2017

RESUMEN: En este artículo pretendemos ofrecer una aproximación acerca de la incorporación de la mujer a los estudios secundarios de los Institutos de Cáceres y Badajoz, conocidos como Institutos Históricos extremeños, en el primer tercio del siglo xx. Principalmente, el foco de interés se ha centrado en conocer la evolución que experimentó esa incorporación de las mujeres a la educación secundaria y las causas que favorecieron o dificultaron su desarrollo. En Extremadura, la evolución de la segunda enseñanza estuvo marcada por el desarrollo socioeconómico de la región, que mostraba unas características muy peculiares, y que fueron determinantes en la estructura social, cultural y económica de la misma. A pesar de ello, estas circunstancias no impidieron el acceso de las mujeres a los estudios de bachillerato.

Palabras Clave: mujeres; educación secundaria; siglo xx; Extremadura.

AвSTRACT: In this article we pretend to offer an approximation about the incorporation of women to secondary studies in the high schools of Caceres and Badajoz, known as historic high schools of Extremadura, in the first of the $20^{\text {th }}$ century. Mainly, the principle point of interest has been the knowledge of the evolution that the incorporation of women to secondary education has experimented and the causes that made good or bad to its development. In Extremadura, the evolution of secondary education was marked by the socioeconomic development of the region, that showed a very peculiar characteristics and that were decisive for the social, 
cultural and economic structure of it. However these circumstances didn't avoid the access of women to high studies.

KEY WORDS: women; Secondary studies; xx century; Extremadura.

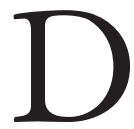

ADO QUE EL PRIMER TERCIO DEL SIGLO XX es una de las etapas más complejas e interesantes de nuestra historia, ha sido y es objeto de múltiples y variados estudios. En el caso concreto de Extremadura, se han publicado valiosos trabajos en los cuales se han abordado temas demográficos, de economía, sobre las agudas tensiones sociales del momento, la dinámica de la política en la crisis de la Restauración y algunas monografías sobre diversos temas culturales y educativos. Sin embargo, dentro de la historiografía educativa de la región extremeña la búsqueda de estudios sobre la segunda enseñanza y, concretamente, acerca de la incorporación de las mujeres a este nivel educativo, centrada sobre todo en estas décadas nos permitió percatarnos de la escasez de trabajos orientados en este sentido; los pobres resultados de la misma acrecentaron considerablemente nuestro interés inicial en el desarrollo de una labor de investigación que sirviese para paliar, en cierta medida, la referida escasez, contribuyendo de paso al enriquecimiento de la propia historiografía educativa de Extremadura.

Con tal fin, este trabajo pretende dar una visión del desarrollo y evolución de la incorporación de las mujeres a la segunda enseñanza pública en Extremadura, concretamente de los Institutos Históricos de la región, el IEs «El Brocense» de Cáceres y el IEs «Bárbara de Braganza» de Badajoz, desde 1900 hasta 1936 ..

La metodología utilizada ha sido la propia del método histórico-educativo, combinando el análisis cualitativo y cuantitativo, que no hubiesen sido posibles sin recurrir a ciertas fuentes documentales. Se ha realizado una consulta pormenorizada de las fuentes primarias, representadas principalmente por las Memorias de los Institutos recopiladas en los siguientes archivos: Archivo General de la Administración (AGA), Archivo Histórico de la Universidad de Sevilla (AUs), Archivo Histórico de la Universidad de Salamanca (AUSA), Archivo Histórico Provincial de Badajoz (AHPB) y Archivo del Instituto «El Brocense» de Cáceres (AIBC). Además, nos han sido muy útiles también las series estadísticas sobre educación y cultura proporcionadas por los Anuarios, aun teniendo en cuenta que a veces los datos son imprecisos o dejan considerables espacios por cubrir; también hemos consultado la Colección Legislativa del Ministerio de Instrucción Pública, de la que hemos extraído las disposiciones referentes a la Educación Secundaria.

El nacimiento y la consolidación de ambos centros han sido ya estudiados por Domínguez Rodríguez, E.: Cáceres y la Enseñanza Secundaria, 1822-1869, Cáceres, Institución Cultural «El Brocense», I99I; Sánchez Pascua, F.: El Instituto de segunda enseñanza en Badajoz en el siglo XIX, Badajoz, Departamento de Publicaciones del Excma. Diputación de Badajoz, 1985; SÁnCHEZ Pascua, F.: Política y educación. Incidencias en el Instituto de segunda enseñanza en Badajoz (I8451900), Badajoz, Universidad de Extremadura, 1985; Sánchez PAscuA, F.: «La enseñanza secundaria extremeña en el tránsito del siglo xIx al xx. Regeneracionismo», Revista de Estudios Extremeños, t. LIV, n. ${ }^{\circ} 2$ (1998) pp. 557-587. 
LA INCORPORACIÓN DE LAS MUJERES A LA SEGUNDA ENSEÑANZA PÚBLICA

EN EXTREMADURA (I900-I936)

CRISTINA REDONDO CASTRO, ISABEL GRANA GIL Y CARMEN SANCHIDRIÁN BLANCO

\section{Las mujeres y la segunda enseñanza ${ }^{2}$}

Desde su configuración, como un nivel del sistema educativo, la segunda enseñanza se constituyó como un periodo de formación destinado a los hijos varones de las clases medias, ya que se concebía como un espacio masculino3. Podríamos hablar entonces de un modelo excluyente, donde las mujeres sólo serían bien recibidas y tendrían su lugar en espacios reservados para ellas, como eran las enseñanzas domésticas o los colegios privados.

La incorporación de la mujer al bachillerato ha estado desde sus inicios, en el siglo XIX, llena de obstáculos, pues tuvieron que enfrentarse a las trabas que el sistema y la sociedad, en general, les iban poniendo para poder acceder a estos estudios.

2 En las últimas décadas se han llevado a cabo numerosos trabajos centrados en las mujeres visibilizando el papel que han desempeñado a lo largo de la historia en la vida política, científica, social y económica. En el tema de estudio, las mujeres en la segunda enseñanza, pueden consultarse, entre otros, los trabajos realizados por Alcázar CRUz Rodríguez, M. ${ }^{a}$ : Historia del Instituto Santísima Trinidad de Baeza (I869-1953). Aportaciones al estudio de la educación de las mujeres, Tesis doctoral, 20oI. Recuperada de http://ruja.ujaen.es/handle/10953/321; Araque HonTANGAs, N.: «El Instituto Femenino Infanta Beatriz y la inserción de las mujeres en los institutos de Enseñanza Secundaria de Madrid (I900-1930)", Revista Complutense de Educación, vol. I2, n. ${ }^{\circ} 2$ (200I), pp. 753-78I; ARAQue Hontangas, N. y Poveda Sanz, M.: «La presencia de las mujeres en la segunda enseñanza de Madrid», en López-Ocón, L.; Aragón, S.; Pedrazuela, M. (eds. lit.) y Juaristi, J. (pr.): Aulas con Memoria: ciencia, educación y patrimonio en los institutos históricos de Madrid (I837-1936), Madrid, ceimes, 20i2, pp. 209-224; Benso Calvo, C. y Cid Galante, R. M.: «Los expedientes de las estudiantes de bachillerato: una fuente para el estudio del alumnado femenino de los institutos. Ourense como ejemplo (1900-1930)», Revista de Historia de la Educación, 26 (2007), pp. 437-470; Capel Martínez, R. M.: «El archivo de la residencia de señoritas», Revista de CEE Participación Educativa, n. ${ }^{\circ}$ ir (julio de 2009), pp. I56-I6r; Capel Martínez, R. M.: «Mujer y educación en el reinado de Alfonso XIII. Análisis cuantitativo", Cuadernos de Historia Moderna y Contemporánea, n. ${ }^{\circ} 2$ (198I), pp. 23I-250; Fernández, P.; Torres, I.; Baselga, C. y Gaudó, C.: «Los bachilleratos femeninos», en VICENTE y GUERRERo (coord. y ed. Tit.): Estudios sobre la historia de la enseñanza media en Aragón, Zaragoza, Instituto Fernando el Católico csic, Excma. Diputación de Zaragoza, 20I2, pp. I09-I46; Flecha García, C.: «La incorporación de las mujeres a los institutos de educación secundaria en España», Revista de Historia de la Educación, núm. 17 (1998), pp. 159-178; FlechA García, C.: «Profesoras y alumnas en los Institutos de Segunda Enseñanza. I9IO-I940», Revista de Educación, núm. extraordinario (2000), pp. 269-294; GonzÁlez PÉrEz, T.: «Las primeras alumnas en los institutos de bachillerato en Canaria", Educació $i$ Història: Revista d'Història de l'Educació, núm. I7 (gener-juny, 20II), pp. IoI-I32; Herrero Sáenz, R.: «La incorporación de las mujeres a la educación secundaria durante la Segunda República: Un estudio de casos sobre el Instituto Quevedo de Madrid», en López-Ocón, L. (ed.): Aulas modernas. Nuevas perspectivas sobre las reformas de la enseñanza secundaria en la época de la JAE (1907-1939), Madrid, Universidad de Carlos III de Madrid, 20I4, pp. 215-248; VÁzquez RAmil, R.: Mujeres y Educación en la España contemporánea. La Institución Libre de Enseñanza y la Residencia de Señoritas de Madrid, Madrid, Akal, 20I2; Verdasco, M. M.: «Alumnas en el instituto de Badajoz I900-1920», en Gómez, M. N. (ed.): Presente, pasado y futuro de la educación secundaria en España, Sevilla, Ed. Kronos, I996; Viñao Frago, A.: «Espacios masculinos, espacios femeninos. El acceso de la mujer al bachillerato», en Mujer y educación en España, 1868-1975. Actas VI Coloquio de Historia de la Educación, Santiago de Compostela, Universidad de Santiago, I990.

3 Viñao Frago, A.: op. cit., p. 567. 
Aunque no existía una prohibición expresa que impidiera el acceso de las mujeres a este nivel educativo, se sobreentendía que no era propio de ellas. Sólo podían asistir a la enseñanza primaria, a los colegios de señoritas y Escuelas Normales y, salvo excepciones, ni ellas se planteaban matricularse en los Institutos ${ }^{4}$. Es decir, desde sus orígenes la enseñanza secundaria tenía marcados sus objetivos y entre ellos no se establecía la formación cultural de la mujer pues ésta no debía alejarse de las pautas sociales establecidas para ella y, en este sentido, la formación recibida tenía que ir encaminada a perfeccionar su misión -buena esposa y madre-, de ahí que se fijaran límites a su educación, como ponen de manifiesto las políticas educativas que fueron conformando la evolución de la enseñanza de la mujer en los estudios secundarioss.

Como recoge Flecha, lo que preocupaba a los hombres era la igualdad intelectual, más que la igualdad política o social ${ }^{6}$. Les asustaba una realidad social en la que cambiaran los consolidados equilibrios en las relaciones entre los sexos. Es por ello que ni pensaban, ni imaginaban, ni esperaban la incorporación de la mujer a los estudios secundarios o universitarios?

Fue en riro cuando se establece el acceso de las mujeres a los estudios secundarios en igualdad con los hombres, aunque para llegar a ese momento tuvieron que superar muchas trabas que legalmente ralentizaron el acceso de la mujer a estas enseñanzas. A finales del siglo xIx, las familias más acomodadas empezaron a reclamar el derecho de que sus hijas pudieran cursar estos estudios; en el año I870 fue cuando la primera mujer solicitó matricularse en el bachillerato y desde ese momento el número de solicitudes iba aumentando ya que cada vez había más mujeres que querían cursar estos estudios. Ante esto, pronto se empezarían a tomar las medidas legales para frenar su entrada. En I882, por Real Orden de I6 de marzo, se prohibió el acceso de la mujer a los estudios universitarios. Posteriormente, por medio de otra Orden en la que se dejaba constancia de que la mujer no tenía cabida en estos estudios, se estableció ya expresamente la «no admisión a matrícula para asignaturas de segunda enseñanza a las Señoritas». Un año después, se autorizaba que las mujeres pudieran cursar estudios secundarios, pero sólo en centros privados, para evitar la confluencia con el sexo masculino. Será a partir de i 888 cuando se dé un pequeño paso adelante pues, mediante petición, las mujeres podían solicitar el acceso a los centros oficiales para cursar el Bachillerato, modelo que estuvo vigente hasta i9io.

Con el cambio de siglo, conservadores y liberales colaboraron por el objetivo común de fomentar la educación pública, inspirándose en el texto de Cossío

Cfr. González PÉRez, T.: op. cit., pp. IOI-I32.

Redondo Castro, C. y Alonso Briales, M.: «Las mujeres y la enseñanza secundaria en el periodo republicano", en Convivencia y resolución de conflictos en contextos socioeducativos. Igualdad de género y educación para la convivencia. Actas II Congreso Internacional y IV Congreso Nacional sobre Convivencia y resolución de conflictos en contextos socioeducativos, Málaga, Universidad de Málaga, 20I5.

6 Flecha García, C.: «Políticas y espacios...», op. cit., p. 83.

7 Ibidem. 
LA INCORPORACIÓN DE LAS MUJERES A LA SEGUNDA ENSEÑANZA PÚBLICA

EN EXTREMADURA (I900-I936)

CRISTINA REDONDO CASTRO, ISABEL GRANA GIL Y CARMEN SANCHIDRIÁN BLANCO

de I899 en el que se recoge «que todas las reformas dichas deben de entenderse igualmente por lo que toca a la educación de la mujer, que no importa separarla de la del hombre» ${ }^{8}$. Como se puede apreciar, los institucionistas ya ponían de manifiesto su preocupación por la situación de la mujer y trabajaron para conseguir acortar la distancia entre la instrucción femenina y masculina.

Una vez puesto en marcha el Ministerio de Instrucción Pública y Bellas Artes, en 1900 , los organismos dependientes del mismo comenzaron a poner en funcionamiento instituciones que favorecieran la instrucción de las mujeres, entre las que cabe destacar el Instituto Escuela y la Residencia de Señoritas.

Poco a poco, el rol asignado por la sociedad a las mujeres iba evolucionando y su formación restringida antes al ámbito del magisterio, de la familia y el hogar, ahora se abría a una formación más amplia que le permitía acceso a otros ámbitos profesionales y sociales, sin embargo, es necesario matizar que en un principio sólo se vieron favorecidas las mujeres de las clases medias y altas. Fue con la II República y a través de sus políticas educativas cuando se posibilitaría la plena incorporación de las mujeres españolas a todos los niveles educativos y especialmente a la enseñanza secundaria, pues para el régimen republicano la educación era un compromiso con la sociedad, cuyo fin era lograr la democratización, garantizar los derechos de todos los ciudadanos y ciudadanas y modernizar el país'. Para ello, proponían una educación obligatoria, gratuita, laica... en la que todos y todas tuvieran acceso sin discriminación por sexo o clase social. Los mensajes difundidos en estos años hicieron que la presencia de las mujeres en este nivel educativo fuera real y que entonces no solo se matricularan para ampliar los conocimientos de la enseñanza primaria, sino que se prepararan para continuar en la universidad ${ }^{10}$. Además, el incremento de matrículas en los Institutos se vio favorecido por la reforma de i93i de las Escuelas Normales, ya que, entre otras cosas, se exigían los estudios de bachillerato para acceder a las mismas, y como sabemos la carrera de maestra era una de las profesiones más aceptadas para las mujeres"

Estos planteamientos fueron los que promovieron el aumento de la presencia de las mujeres en las aulas de los institutos en el primer tercio del siglo xx. Nuestro siguiente objetivo será estudiar cómo se reflejaron las nuevas posibilidades que ofrecía el sistema educativo en los institutos extremeños.

8 Ministerio de Educación: Historia de la Educación en España, tomo iit. Madrid, mec, 1982, p. 347 .

9 Sánchez de Madariaga, E. (ed.): Las maestras de la República, Madrid, Catarata, 20I2, p. 8.

1o Flecha García, C.: «La segunda república, las mujeres y la educación», en SÁnchez DE Madariaga, E. (ed.): op. cit., p. 43.

II La exigencia del título de bachillerato para acceder a las Escuelas Normales supuso un descenso en el número de matrículas en las mismas, pues quien decidía cursar los estudios secundarios lo hacían con miras a seguir carreras universitarias. Para saber más cfr. Flecha García, C: «La segunda república...», op. cit., pp. 48-49. 


\section{La realidad cultural extremeña}

La cultura popular que hasta el momento había estado en un segundo plano, con el inicio del nuevo siglo, pasa a ser una de las grandes preocupaciones del Estado haciéndose responsable de la misma y reclamando los derechos y el deber de proporcionar a los ciudadanos una instrucción adecuada, aunque las medidas tomadas fueron insuficientes.

Según datos del censo de 1912 la población total de «hecho» de España en I900 era de I8.618.086 habitantes de los cuales no sabían leer ni escribir ir.874.89o, sabían leer 495.753, sabían leer y escribir 6.227.184 y 20.259 no constan, lo cual suponía que un $63,78 \%$ de la población total no sabía ni leer ni escribir.

En el caso de Extremadura, a principios de siglo nos encontramos con una región abandonada en muchos aspectos. Si tenemos en cuenta los agentes externos que determinan la evolución de la educación de la región durante el primer tercio del siglo xx vemos una comunidad subdesarrollada: elevada tasa de nacimientos y defunciones, sobre todo por infecciones y parasitismos, siendo las tasas más elevadas las asociadas a la mortalidad infantil; fuerte crecimiento demográfico a pesar de la pérdida de población, que emigra a las grandes ciudades en busca de trabajo, al recibir población inmigrante que viene a ocupar los puestos que estos dejan; un gran desequilibrio entre los diferentes sectores económicos, con un predominio del sector agrario y elevado índice de analfabetismo, sobre todo femenino.

El número de personas que no sabían leer y escribir era muy elevado, así en I90o un $74 \%$ de la población extremeña era analfabeta, siendo el mayor índice de analfabetismo el de la población femenina, que representaba un 81,3\%. En I9ıо el porcentaje de analfabetos descendía a un 68\%. En i930 el analfabetismo había descendido en dieciocho puntos respecto a 1900 , pasando a un $55,5 \%$ de la población; aun así, seguía siendo un porcentaje muy elevado, pues más de la mitad de la población extremeña no sabía ni leer ni escribir, aunque observamos la tendencia de mejora.

Atendiendo a la desigualdad entre géneros, y pese a la disminución progresiva del índice de analfabetismo, resulta alarmante comprobar que éste sigue siendo más elevado entre las mujeres, pues en 1940 alcanzaba el 50\%, es decir, que más de la mitad de la población femenina seguía sin saber leer y escribir.

Estos datos nos muestran que el nivel cultural de la sociedad, y en especial el de la mujer, seguía siendo muy deficiente. En el caso de Extremadura, podría deducirse que las medidas adoptadas, escuelas y maestros, fueron insuficientes, aunque no pueden negarse los avances en la instrucción elemental de la población, ya que el índice de analfabetismo desciende de un 74,1\% en 1900 a un 47,1\% en 1940 . En cuarenta años el analfabetismo había descendido en más de veinte puntos.

$\mathrm{Si}$ estos datos los comparamos con los nacionales obtenemos que en 1900 en España el porcentaje de hombres analfabetos era del $55,78 \%$ mientras que el de las mujeres se elevaba al 71,43\%. En 1940 las cifras respectivamente eran $29,66 \%$ y $37,73 \%$. 
LA INCORPORACIÓN DE LAS MUJERES A LA SEGUNDA ENSEÑANZA PÚBLICA

EN EXTREMADURA (I900-I936)

CRISTINA REDONDO CASTRO, ISABEL GRANA GIL Y CARMEN SANCHIDRIÁN BLANCO

Los datos aportados sobre la comunidad extremeña son válidos para sus dos provincias. Es decir, el analfabetismo desciende progresivamente en Badajoz y en Cáceres. En Badajoz se pasa del $76,8 \%$ en 1900 a un $46,8 \%$ en 1940 y en Cáceres las cifras son, en las mismas fechas, del $70,2 \%$ al $47,5 \%$ respectivamente. El descenso del analfabetismo, como podemos observar, fue menos acusado en la provincia de Cáceres.

TABLA I: Distribución de analfabetismo por provincias (I860-1940)

BADAJOZ

\begin{tabular}{|c|c|c|c|c|c|c|c|c|c|}
\hline & \multicolumn{3}{|c|}{ POBLACIÓN DE «HECHO» } & \multicolumn{6}{|c|}{ PoBLACión ANALFABETA } \\
\hline$A \tilde{n} o$ & Hombres & Mujeres & Total & Varones & $\%$ & Mujeres & $\%$ & Total & $\%$ \\
\hline I9IO & 298.193 & 295.013 & 593.206 & I9I.I25 & $60, \mathrm{I}$ & 219.074 & 74,3 & 410.199 & 69,1 \\
\hline 1920 & 324.232 & $32 \mathrm{I} .426$ & 644.625 & 200.086 & $6 \mathrm{I}, 7$ & 230.868 & 71.8 & 430.954 & 66,8 \\
\hline 1930 & 350.954 & $35 \mathrm{I} .464$ & 702.416 & 179.442 & $5 \mathrm{I}, \mathrm{I}$ & 220.003 & 62.6 & 399.445 & 59,9 \\
\hline 1940 & 358.003 & 384.544 & 742.547 & 151.042 & 42.2 & 196.565 & $5 \mathrm{I}, \mathrm{I}$ & 347.607 & 46,8 \\
\hline
\end{tabular}

CÁCERES

\begin{tabular}{|c|c|c|c|c|c|c|c|c|c|}
\hline & \multicolumn{3}{|c|}{ Población DE «HECHO» } & \multicolumn{6}{|c|}{ POBLACiÓN ANALFABETA } \\
\hline$A \tilde{n} o$ & Hombres & Mujeres & Total & Varones & $\%$ & Mujeres & $\%$ & Total & $\%$ \\
\hline I9IO & 197.196 & 200.589 & 397.785 & $\mathrm{II} 7.289$ & 59.5 & 150.221 & 74.9 & $267.5 \mathrm{IO}$ & 67.2 \\
\hline 1920 & 202.252 & 207.780 & 410.032 & I08.728 & 53.8 & I46.I4I & 70.3 & 254.869 & 62.2 \\
\hline 1930 & 222.585 & 226.240 & 449.756 & 100.758 & $45 \cdot 3$ & $\mathrm{I} 39.406$ & 6I.I & 240.164 & 53.4 \\
\hline 1940 & 249.206 & $262.17 \mathrm{I}$ & $5 \mathrm{II} .377$ & $97.79 \mathrm{I}$ & 39.2 & $\mathrm{I} 44.889$ & 55.3 & 242.680 & 47.5 \\
\hline
\end{tabular}

Fuente: Anuarios Estadísticos de I900 a 1920/Anuario I9I2 y I920; de I92I a I930/Anuario I930; de I940 a I95I/Anuario 1943.

En el caso de Extremadura, Rodillo ${ }^{12}$ recoge que las causas del alto índice del analfabetismo en la región se debían, primero, al insuficiente número de escuelas para la población existente. Aunque habían aumentado a lo largo del siglo hasta ubicarse una en casi cada localidad, no bastaban para la demanda de la población en edad escolar, siendo inferiores a las que la propia normativa exigía en cada lugar.

Un ejemplo ilustrativo de la situación de la enseñanza elemental en la región extremeña es que en I880 había 843 escuelas públicas y 138 privadas, lo que suponía que a cada escuela le correspondían 774 habitantes, mientras que la media nacional se situaba en 56r habitantes por escuela. En I908 había en la región I.030 escuelas (públicas y privadas) con 94I habitantes por escuela, cifra muy alejada de la nacional ${ }^{13}$. Luis Bello muestra una panorámica sobre la situación de abandono

${ }_{12}$ Rodillo Cordero, F. J.: Datos para la Historia Escolar de Extremadura, Mérida, Editora Regional de Extremadura, I998, pp. II5-II7.

${ }^{13}$ García Pérez, J.; Sánchez Mayorro, F. y Merino Martín, M. J.: Historia de Extremadura. Los tiempos actuales, Badajoz, Universitas, Consejería de Educación y Cultura. Junta de Extremadura, t. IV, 1985, p. 972. 
de la enseñanza primaria en Extremadura, sin dudar en calificar las escuelas como las peores del país, recogiendo que en Cáceres «no hay ni una sola escuela digna de este nombre», las existentes «no son escuelas sino corredores, cuadras de planta baja, húmedas y frías, patio carcelario» ${ }^{14}$. En definitiva, no sólo son escasas sino que, además, se encontraban en lamentables condiciones.

Ejemplo de ello es la descripción que Bohoyo Velázquez hace de la situación de la localidad de Montijo en I902:

Las escuelas Municipales de niñas se hallan instaladas en una casa particular [...] la planta baja, que tiene habitaciones espaciosas la ocupa la profesora con su familia, y la escuela radica en los graneros [...] Se encuentra a teja vana, siendo la techumbre tan baja, que por la parte de la calle se da en ella la mano; por ventilación tiene tres huecos bastante reducidos, todos a la parte norte. El número de niñas es de 238 [...]. Con arreglo al número de habitantes que cuenta esta población, debieran existir, cuando menos, cuatro escuelas de instrucción primaria, sostenidas con fondos públicos ${ }^{15}$.

La segunda causa indicada por Rodillo es el absentismo generalizado. En unos casos el absentismo era forzado por la inexistencia de plazas escolares, en otros por la escasa atracción que la escuela ejercía sobre niños y padres, más interesados éstos en utilizar a sus hijos como mano de obra que en una instrucción cuyas virtualidades no siempre apreciaban.

A pesar de la obligatoriedad establecida por ley, ésta no supuso un incentivo para la asistencia. Los datos recogidos por el Anuario Estadístico de 1912 confirman este hecho. En 1908, en la provincia de Badajoz, de los 58.724 niños en edad escolar, no estaban escolarizados 24.632 , pero hay que tener en cuenta que el hecho de que los niños estuvieran matriculados no significaba que asistieran a la escuela.

La tercera causa son las insuficientes dotaciones oficiales, de los ayuntamientos cuando las escuelas dependían de ellos o del Gobierno estatal cuando pasaron a éste.

La cuarta es la falta de gratificación adecuada a los maestros, por no decir abandono en cuanto a salario, formación y estabilidad. Los maestros estaban mal remunerados y peor considerados. Hasta igir no se les reconoció un sueldo mínimo de I.000 pesetas por año, que en I9I8 fue elevado a I.500 y en I923 a 2.000. La República dignificó aún más la profesión elevando el sueldo a 3.000 pesetas. Todo ello fue posible gracias a la creación del Ministerio de Instrucción Pública en 1900 , con el que se inicia un proceso de extensión y fortalecimiento organizativo de la enseñanza y se consolida la tendencia centralista y jerárquica del sistema educativo ${ }^{16}$. El mismo se encargó del pago de los maestros mediante el traspaso

${ }^{14}$ Bello Trompeta, L.: Viaje por las escuelas de España. Extremadura, Madrid, Espasa-Calpe, 1927.

is Bohoyo Velázquez, I.: Situación socioeconómica y condiciones de vida en la provincia de Badajoz (I880-I902), Badajoz, Editorial Universitas, I984, p. 72.

${ }_{16}$ Mártín Zúñiga, F.: La enseñanza primaria en Málaga durante el reinado de Alfonso XIII (I902-193I). Historia de un atraso endémico, Málaga, Biblioteca Popular Malagueña, I993, pp. 20-22. 
LA INCORPORACIÓN DE LAS MUJERES A LA SEGUNDA ENSEÑANZA PÚBLICA

EN EXTREMADURA (I900-I936)

CRISTINA REDONDO CASTRO, ISABEL GRANA GIL Y CARMEN SANCHIDRIÁN BLANCO

del Tesoro público a los fondos municipales, esto fue regulado por el R. D. de 2I de julio de 1900 . A partir de 1902 el Estado se hizo cargo del sostenimiento de los docentes primarios, que hasta entonces dependían de las áreas municipales, lo que hacía que cobrasen poco y de manera irregular.

Y la quinta y última causa es la estrecha relación encontrada entre analfabetismo y latifundismo $0^{17}$.

En definitiva, a los atrasos regionales en otros aspectos se unían también el atraso educativo y cultural.

A pesar de las medidas tomadas por los diferentes Gobiernos, en materia educativa, los datos del primer tercio del siglo xx eran aun devastadores en cuanto a la instrucción primaria, y, por tanto, cabe preguntarnos, ¿cómo se vieron reflejados estos datos en la segunda enseñanza extremeña?

\section{Las mujeres en los institutos extremeños (1900-1936)}

La evolución de la segunda enseñanza en Extremadura durante el primer tercio del siglo xx vino marcada por el desarrollo socioeconómico de la región. Como hemos visto, esta región mostraba unas características muy peculiares, que en gran medida fueron determinantes para la estructura social, cultural y económica de la misma.

El desarrollo de la segunda enseñanza a lo largo del primer tercio del siglo $\mathrm{xx}$ fue progresivo. Mientras que los Institutos de Cáceres y de Badajoz ${ }^{18}$ iban asentando sus bases y tomando fuerza, otros centros abrirían sus puertas promovidos por las diferentes políticas del momento. En la provincia de Badajoz, durante la Dictadura de Primo de Rivera, comenzaron su andadura los institutos de Zafra y Fregenal de la Sierra y durante la II República los de Villafranca de los Barros y Mérida, al igual que ocurrió con el de Plasencia en la provincia de Cáceres.

Sin embargo, en este trabajo no estudiamos la situación de los diferentes centros encargados de impartir la segunda enseñanza, sino que nos centramos en la incorporación de las mujeres a la educación secundaria en las aulas de los conocidos como Institutos Históricos de la región extremeña.

${ }_{17}$ Este factor ha sido abordado por diversos autores, para saber más puede consultarse la obra de Guzmán Reina, A. y otros: Valoración del analfabetismo en España, Madrid, JNcA, 1955.

${ }_{18}$ La segunda enseñanza como tal, como nivel educativo dentro del sistema educativo, queda configurada en el periodo comprendido entre I837-1857, siendo la Ley Moyano de I857 la expresión legal definitiva de la misma como señala VIÑAO (1982). Este autor diferencia dos fases, la primera de ellas de I837 a I845 en la que aparece la «nueva» educación secundaria y se fijan las bases económicas y legislativas, momento en el que el Instituto de Cáceres abrió sus puertas (I839); y una segunda fase (1845-1857) de consolidación, donde se instaura definitivamente dicho nivel, así como los planes de estudios. Siendo en esta segunda fase donde se consolidaron las bases para la creación del Instituto de Badajoz (I849). Para saber más acerca de la consolidación y desarrollo de la segunda puede $c f r$. Viñao Frago, A.: Política y educación en los orígenes de la España contemporánea. Examen especial de sus relaciones en la enseñanza secundaria, Madrid, Siglo XXI, 1982. 
Para ello, vamos a basarnos fundamentalmente en los Anuarios Estadísticos y las Memorias ${ }^{19}$ de ambos centros. En este sentido, lo primero que llama la atención a la hora de analizar los datos recogidos de los Anuarios Estadísticos es que son irregulares. Algunos datos no figuran todos los años, o se recogen de manera diferente de un curso para otro, o incluso faltan datos en años concretos, por lo que la labor de interpretación se dificulta. Sin embargo, estas limitaciones no nos impiden su lectura mostrando una visión general sobre la incorporación de las mujeres a la educación secundaria pública en Extremadura y las transformaciones más importantes que se llevaron a cabo en la misma.

\section{I. Las alumnas en el Instituto de Cáceres}

En el Instituto de Cáceres la presencia femenina se hizo esperar. No sería hasta las primeras décadas del siglo xx cuando, tímidamente, comenzara a incorporarse la mujer cacereña a los estudios de segunda enseñanza tímidamente.

Como podemos observar en los Gráficos i y II, la evolución en el número de alumnos matriculados en la enseñanza secundaria en la provincia de Cáceres, tanto en la oficial como en la no oficial ${ }^{20}$, fue progresiva aunque no constante. Desde I9I4 hasta 1923, cuando comienza la Dictadura de Primo de Rivera, el número de matrículas fue creciendo progresivamente, siendo en esta última fecha cuando se dispara. En la enseñanza oficial, se pasó de 162 matrículas en el curso de 1922-23 a 626 al curso siguiente, llegando a igualar en número a la enseñanza no oficial. Sin embargo, un año después el número descendía, siendo en la enseñanza oficial donde se aprecia una bajada muy considerable. Aunque los datos comenzaron a ser muy alentadores, vemos un pequeño retroceso en el año de 1926, con la instauración del nuevo plan de estudios, el Plan Callejo, el cual dividía estos estudios en bachillerato elemental y universitario, y en los últimos dos cursos se bifurcaba entre ciencias y letras. Aunque este plan estaba sustentado en contenidos realistas y prácticos, no fue muy bien aceptado, por ser de la Dictadura, y no será hasta la instauración de la II República cuando de nuevo se vuelvan a apreciar aumentos muy significativos.

19 Como se podrá apreciar a lo largo de la exposición, las fechas para el análisis de ambos Institutos no son las mismas, ello se debe a que no hemos podido localizar algunas Memorias y aunque, en ocasiones, hemos intentado completar los datos con los de los Anuarios no siempre ha sido posible.

${ }_{20}$ Cuando hablamos de enseñanza oficial nos referimos al Instituto General y Técnico de Cáceres mientras que, cuando nos referimos a enseñanza no oficial, es a la impartida en los colegios inscritos al Instituto o, lo que es lo mismo, a los colegios privados. En ocasiones, esta última integra datos del alumnado de los colegios privados y de aquellos que se presentaban en modalidad libre. 
LA INCORPORACIÓN DE LAS MUJERES A LA SEGUNDA ENSEÑANZA PÚBLICA

EN EXTREMADURA (I900-I936)

CRISTINA REDONDO CASTRO, ISABEL GRANA GIL Y CARMEN SANCHIDRIÁN BLANCO

GráfIco I: Alumnos matriculados en la enseñanza oficial, Cáceres (I9I4-1933)21

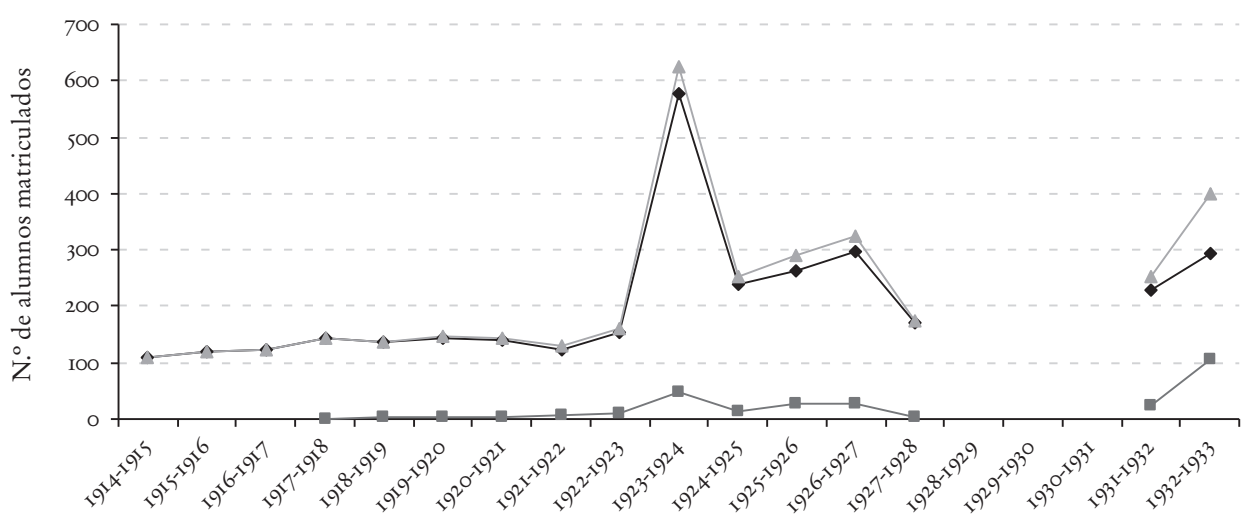

Cursos académicos

$\multimap$ Hombres $\rightarrow-$ Mujeres $\longrightarrow$ Total

Fuente: Elaboración propia a partir de los datos del Ine. Anuarios Estadísticos de 1915 a 1934.

Gráfico II: Alumnas matriculadas en la enseñanza oficial y no oficial (I9I4-I933)

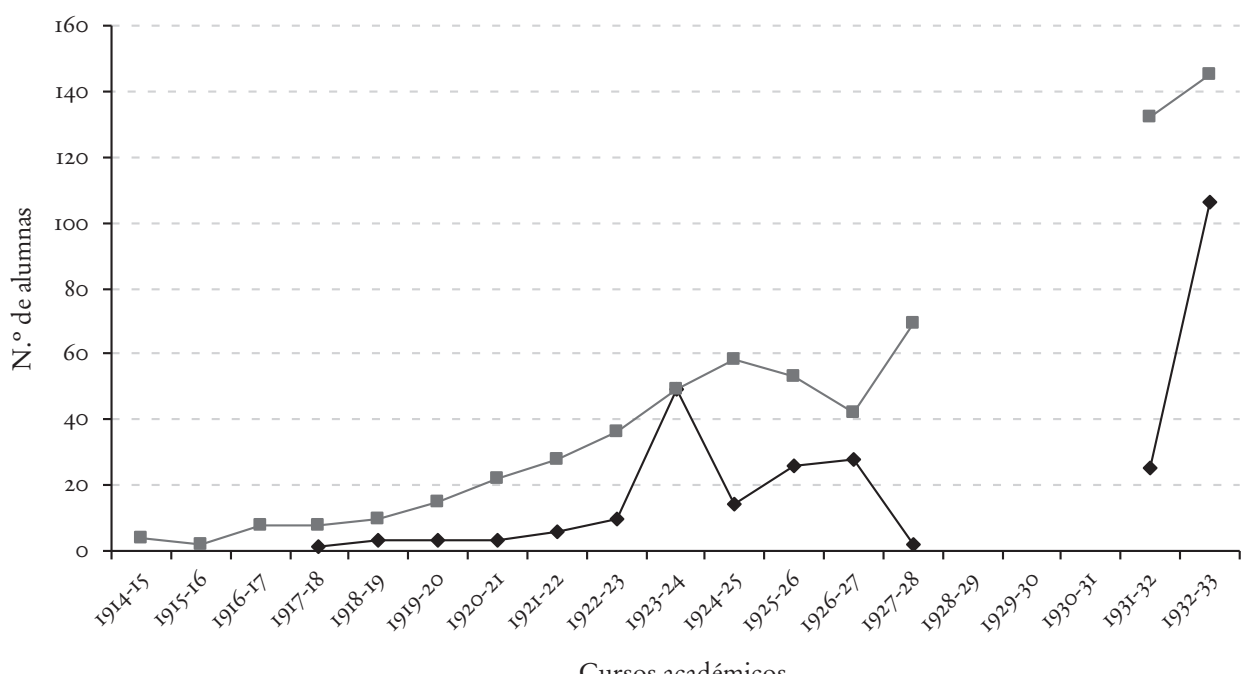

Cursos académicos

$\multimap$ Enseñanza oficial $\longrightarrow$ Enseñanza no oficial

Fuente: Elaboración propia a partir de los datos del Ine. Anuarios Estadísticos de I9I5 a 1934.

${ }^{21}$ Los datos del Gráfico I pueden ampliarse con la información que nos proporcionan las $M e$ morias del Instituto desde 1934 a 1937, pero, al no poseer la misma información para el de Badajoz, hemos optado por acotarlos. Con los datos aportados ya puede apreciarse que durante el primer 
Ya hemos visto que las mujeres no tuvieron fácil el acceso a la segunda enseñanza, y que hasta igio no se les reconoce el derecho pleno de acceder a estos estudios en igualdad de condiciones que los hombres. Sin embargo, como podemos apreciar en el Gráfico i, la incorporación de la mujer cacereña a los estudios secundarios fue muy tímida, sobre todo en la enseñanza oficial, pues aproximadamente un 71\% de las chicas optó por cursar el bachillerato a través de la enseñanza no oficial, acogiéndose en su mayoría a la modalidad libre.

La evolución en el número de las matrículas de las alumnas fue paralela a la evolución del número de matrículas totales, por lo que se aprecia un auge en el número de éstas en el año 1923, muy pronunciado en la enseñanza oficial. A pesar de que, como comentábamos anteriormente, al año siguiente cayó considerablemente, el número de matrículas aumentó respecto a cursos anteriores. Entre las pretensiones de las políticas educativas del régimen dictatorial se encontraba la modernización del sistema y se entendió que este nivel educativo debía ser un medio a través del cual se pudiera obtener una cultura general, y de esta manera se ayudó a romper con la educación elitista y se promovió el acceso de las clases medias y de las mujeres. Además, para poder acceder a la Formación Profesional era necesario estar en posesión del título de bachiller elemental, por lo que, quizás, aumentaron las matrículas.

Pero, cuando más claramente se aprecia la influencia de las políticas en el creciente número de alumnas matriculadas fue en el periodo republicano. Desde el inicio de la II República hasta 1937, el número de matrículas femeninas se multiplicó por io en la enseñanza oficial, mientras que en la no oficial se mantuvo más constante, situándose por debajo de la enseñanza oficial ${ }^{22}$.

Sin embargo, en general la presencia femenina en este Instituto provincial fue escasa, no siendo hasta 1932 cuando se aprecia el dato más significativo, Io6 mujeres matriculadas, lo que suponía un $26,6 \%$ del total. Estos datos nos muestran que el ideario educativo republicano se hizo patente y favoreció no sólo el acceso de las mujeres a los estudios secundarios, sino que favoreció el acceso a estos estudios de un mayor número de ciudadanos.

Estos datos nos revelan una dura realidad y es que, pese a que el número de alumnas fue incrementándose, aún continuaba siendo bajo porque seguían pesando las concepciones tradicionales ${ }^{23}$ y porque este tipo de cambios necesitan siempre su tiempo.

Factores como el bajo nivel económico y cultural, así como la resistencia de las familias, la opinión dominante, los prejuicios sociales y la falta de concienciación femenina contribuyeron a que el proceso de matriculación femenina en los centros

bienio republicano se da un auge en el número de matrículas, dinámica que siguió durante todo el periodo, así en el curso de $1935-36$ el número de alumnas matriculadas en la enseñanza oficial ascendió a un 32,1\%. Aunque no tenemos datos del Instituto de Badajoz podemos sobreentender que esta dinámica también se dio en el Instituto pacense.

${ }^{22}$ En el curso de 1936-37 el número de matrículas femeninas ascendía a 277 en la enseñanza oficial y a I42 en la no oficial.

${ }_{23}$ Cfr. González Pérez, T.: op. cit., p. I26. 
LA INCORPORACIÓN DE LAS MUJERES A LA SEGUNDA ENSEÑANZA PÚBLICA

EN EXTREMADURA (I900-I936)

CRISTINA REDONDO CASTRO, ISABEL GRANA GIL Y CARMEN SANCHIDRIÁN BLANCO

de educación secundaria no terminara de cuajar, pero ayudó a abrir un camino esperanzador a muchas mujeres y a plantear la reorientación de la educación femenina, tendente a hacer compatible la responsabilidad doméstica derivada de «su naturaleza» con el cultivo intelectual y el ejercicio de la profesión elegida ${ }^{24}$.

TABLA II: Alumnado matriculado en el Instituto de Cáceres (I9I4-1933)

\begin{tabular}{|c|c|c|c|c|c|c|c|c|c|c|}
\hline \multirow{3}{*}{$\begin{array}{c}\text { AÑOS } \\
\text { DE LOS CURSOS } \\
\text { ESCOLARES }\end{array}$} & \multicolumn{10}{|c|}{ ALUMNADO MATRICULADO } \\
\hline & \multicolumn{5}{|c|}{ ENSEÑANZA OFICIAL } & \multicolumn{5}{|c|}{$\begin{array}{c}\text { ENSEÑANZA NO OFICIAL } \\
\text { Colegiada y libre }\end{array}$} \\
\hline & Hombres & $\%$ & Mujeres & $\%$ & Total & Hombres & $\%$ & Mujeres & $\%$ & Total \\
\hline I9I4-I9I5 & I09 & IOO & - & - & IO9 & 188 & 97,9 & 4 & $2, \mathrm{I}$ & 192 \\
\hline I9I5-I9I6 & II8 & $\mathrm{IOO}$ & - & - & II8 & $22 \mathrm{I}$ & $99, \mathrm{I}$ & 2 & 0,9 & 223 \\
\hline I916-I9I7 & $\mathrm{I} 24$ & IOO & - & - & $\mathrm{I} 24$ & $27 \mathrm{I}$ & $97, \mathrm{I}$ & 8 & 2,9 & 279 \\
\hline I917-I918 & $\mathrm{I} 43$ & 99,3 & $\mathrm{I}$ & 0,6 & I44 & 274 & 97,2 & 8 & 2,8 & 282 \\
\hline I918-I919 & 135 & 97,8 & 3 & 2,2 & 138 & 276 & 96,5 & IO & 3,5 & 286 \\
\hline 1919-1920 & $\mathrm{I} 44$ & 98 & 3 & 2 & I47 & 265 & 94,4 & 15 & 5,6 & 280 \\
\hline I920-I92I & I4I & 97,9 & 3 & $2, \mathrm{I}$ & I44 & $32 \mathrm{I}$ & 93,6 & 22 & 6,4 & 343 \\
\hline I92I-I922 & 123 & 95,3 & 6 & 4,7 & $\mathrm{I} 29$ & $36 \mathrm{I}$ & 92,8 & 28 & 7,2 & 389 \\
\hline I922-I923 & 152 & 93,8 & IO & 6,2 & $\mathrm{I} 62$ & 362 & 99 & 36 & 9 & 398 \\
\hline I923-I924 & 577 & 92,2 & 49 & 7,8 & 626 & 577 & 92,2 & 49 & 7,8 & 626 \\
\hline $1924-1925$ & 240 & 94,5 & $\mathrm{I} 4$ & 5,5 & 254 & 507 & 89,7 & 58 & IO,3 & 565 \\
\hline $1925-1926$ & 264 & $9 \mathrm{I}$ & 26 & 9 & 290 & 570 & $9 \mathrm{I}, 5$ & 53 & 8,5 & 623 \\
\hline $1926-1927$ & 297 & $9 \mathrm{I}, 4$ & 28 & 8,6 & 325 & 447 & $9 \mathrm{I}, 4$ & 42 & 8,6 & 489 \\
\hline I927-I928 & $\mathrm{I} 7 \mathrm{I}$ & 98,8 & 2 & $\mathrm{I}, 2$ & 173 & $45 \mathrm{I}$ & 86,7 & 69 & $\mathrm{I} 3,3$ & 520 \\
\hline $1928-1929$ & - & - & - & - & - & - & - & - & - & - \\
\hline I929-1930 & - & - & - & - & - & - & - & - & - & - \\
\hline I930-I93I & - & - & - & - & - & - & - & - & - & - \\
\hline I93I-I932 & 228 & $90, \mathrm{I}$ & 25 & 9,9 & 253 & $46 \mathrm{I}$ & 77,7 & $\mathrm{I} 32$ & 22,3 & 593 \\
\hline I932-I933 & 292 & 73,4 & 106 & 26,6 & 398 & $48 \mathrm{I}$ & 76,8 & $\mathrm{I} 45$ & $2 \mathrm{I}, 2$ & 626 \\
\hline
\end{tabular}

Fuente: Elaboración propia a partir de los datos del INE. Anuarios Estadísticos de 1915 a 1934.

\subsection{Las alumnas en el Instituto de Badajoz}

En el instituto de Badajoz, a diferencia del de Cáceres, las primeras matrículas de alumnas se producen en las últimas décadas del siglo xix, como ya venía sucediendo en otras provincias. Hasta que finaliza el siglo, siete fueron las mujeres que iniciaron los estudios de segunda enseñanza, aunque sólo una consiguió el título de bachiller25.

${ }_{24}$ Ruiz DE AzÚA, E.: «Un primer balance de la educación en España en el siglo XX», Cuadernos de Historia Contemporánea, núm. 22 (2000), p. 174.

${ }_{25}$ Sánchez Pascua, F.: El Instituto de Segunda Enseñanza en Badajoz..., op. cit., p. 155 . 
El comienzo del nuevo siglo, como señala Verdasco en su estudio acerca de las alumnas que consiguieron obtener el título de bachillerato en las fechas comprendidas entre 1900 a $192 \mathrm{O}^{26}$, aunque no supuso un gran avance para la educación femenina, significó un antes y un después en este tema. La evolución del acceso de las mujeres al bachillerato sufrió un despertar en las primeras décadas del siglo xx, que se frenaría con el franquismo, aunque el acceso de chicas a este nivel siguió subiendo. Este autor nos muestra que sólo veinte alumnas consiguieron acabar sus estudios secundarios y obtener el título de bachiller. Sin embargo, a pesar de que pocas finalizasen sus estudios, muchas fueron las que, poco a poco, accedieron al instituto para cursar asignaturas sueltas o para hacer el bachiller, aunque esto último era más excepcional. En las primeras décadas la incorporación de las chicas al Instituto de Badajoz fue muy tímida. En I9I4, aproximadamente, un $2 \%$ del total del alumnado matriculado (enseñanza oficial y no oficial) era femenino. Como puede observarse en la Tabla III, el acceso de las mujeres iba ascendiendo progresivamente, llegando a representar un $24 \%$ aproximadamente, en el curso de $1932-33$.

TABLA III: Alumnado matriculado en el Instituto de Badajoz (I9I4-I933)

\begin{tabular}{|c|c|c|c|c|c|c|c|c|c|c|}
\hline \multirow{3}{*}{$\begin{array}{c}\text { ANONOS } \\
\text { DE LOS CURSOS } \\
\text { ESCOLARES }\end{array}$} & \multicolumn{10}{|c|}{ ALUMNADO MATRICULADO } \\
\hline & \multicolumn{5}{|c|}{ ENSEÑANZA OFICIAL } & \multicolumn{5}{|c|}{$\begin{array}{c}\text { ENSEÑANZA NO OFICIAL } \\
\text { Colegiada y libre }\end{array}$} \\
\hline & Hombres & $\%$ & Mujeres & $\%$ & Total & Hombres & $\%$ & Mujeres & $\%$ & Total \\
\hline I9I4-I9I5 & 201 & 97,6 & 5 & 2,4 & 206 & 754 & 97,7 & $\mathrm{I} 3$ & 2,3 & 767 \\
\hline $1915-1916$ & 212 & 97,2 & 6 & 2,8 & 218 & 826 & 98,2 & 15 & $\mathrm{I}, 8$ & $84 \mathrm{I}$ \\
\hline I9I6-I917 & 187 & 96,4 & 7 & 3,6 & 194 & 888 & 98,2 & 16 & $\mathrm{I}, 8$ & 904 \\
\hline I9I7-I9I8 & 217 & 96,4 & 8 & 3.6 & 225 & 886 & 98,8 & II & $\mathrm{I}, 2$ & 897 \\
\hline I918-1919 & 225 & 98,7 & 3 & $\mathrm{I}, 3$ & 228 & 952 & 97,5 & 24 & 2,5 & 976 \\
\hline I919-1920 & 209 & 98,6 & 3 & $\mathrm{I}, 4$ & $2 \mathrm{I} 2$ & 986 & 97,4 & 26 & 2,6 & I.OI2 \\
\hline I920-I92I & 233 & 95,9 & IO & $4, \mathrm{I}$ & 243 & $87 \mathrm{I}$ & 96,6 & $3 \mathrm{I}$ & 3,4 & 902 \\
\hline I92I-I922 & $26 \mathrm{I}$ & 95,6 & $\mathrm{I} 2$ & 4,4 & 273 & 870 & 96 & 36 & 4 & 906 \\
\hline I922-I923 & 262 & 95,3 & 13 & 4,7 & 275 & 903 & 93,8 & 60 & 6,2 & 963 \\
\hline I923-I924 & 280 & 92,7 & 22 & 7,3 & 302 & 926 & 93,5 & 64 & 6,5 & 990 \\
\hline 1924-1925 & 319 & 93 & 24 & 7 & 343 & 920 & 90,5 & 97 & 9,5 & I.OI7 \\
\hline $1925-1926$ & 368 & 92,2 & $3 \mathrm{I}$ & 7,8 & 399 & IOOO & $90, \mathrm{I}$ & IIO & 9,9 & I.IIO \\
\hline I926-I927 & 372 & 88,6 & 48 & II,4 & 420 & 1.057 & 92,2 & 80 & 7,8 & I.I47 \\
\hline I927-I928 & 254 & 87,3 & 37 & $\mathrm{I} 2,7$ & $29 \mathrm{I}$ & 884 & 93,4 & 62 & 6,6 & 946 \\
\hline $1928-1929$ & - & - & - & - & - & - & - & - & - & - \\
\hline I929-1930 & - & - & - & - & - & - & - & - & - & - \\
\hline I930-I93I & - & - & - & - & - & - & - & - & - & - \\
\hline I93I-I932 & 304 & $83, \mathrm{I}$ & 62 & 16,9 & 366 & I.I83 & 80,7 & 283 & 19,3 & I. 466 \\
\hline I932-I933 & 432 & 76,2 & I35 & 23,8 & 567 & I.I63 & 79,3 & 303 & 20,7 & I. 466 \\
\hline
\end{tabular}

Fuente: Elaboración propia a partir de los datos del INE. Anuarios Estadísticos de 1915 a 1934.

26 Verdasco Martín, M. ${ }^{a}$ M.: op. cit., pp. 175-182. 
LA INCORPORACIÓN DE LAS MUJERES A LA SEGUNDA ENSEÑANZA PÚBLICA

EN EXTREMADURA (I900-I936)

CRISTINA REDONDO CASTRO, ISABEL GRANA GIL Y CARMEN SANCHIDRIÁN BLANCO

Gráfico III: Alumnos matriculados en la enseñanza oficial, Badajoz (19I4-I933)

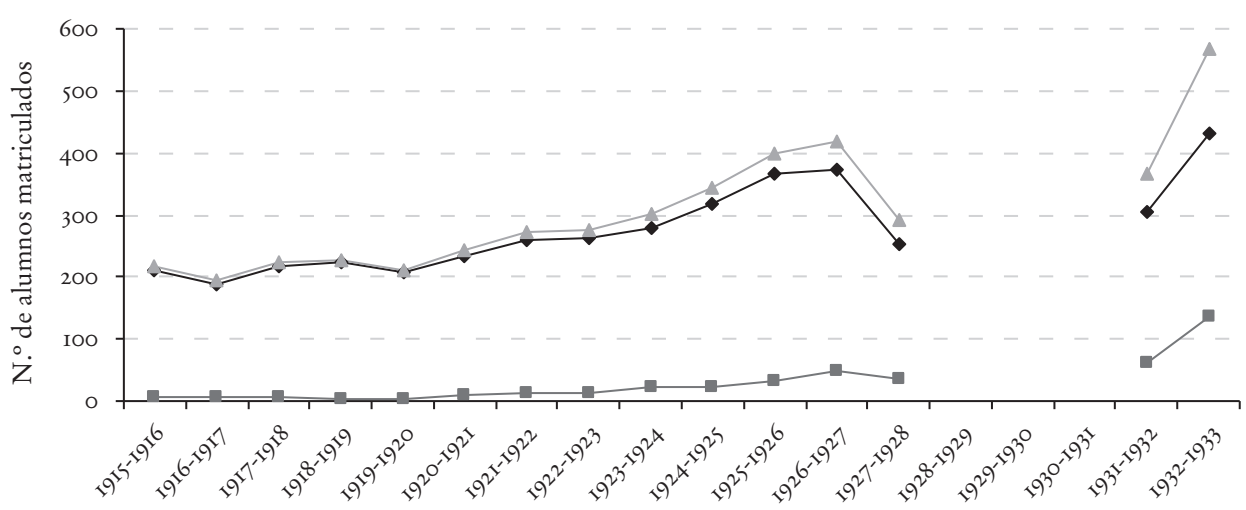

Cursos académicos

$\multimap$ Hombres $\rightarrow-$ Mujeres $\longrightarrow$ Total

Fuente: Elaboración propia a partir de los datos del ine. Anuarios Estadísticos de I9I5 a 1934.

GrÁfICO IV: Alumnas matriculadas en la enseñanza oficial y no oficial (I9I4-I933)

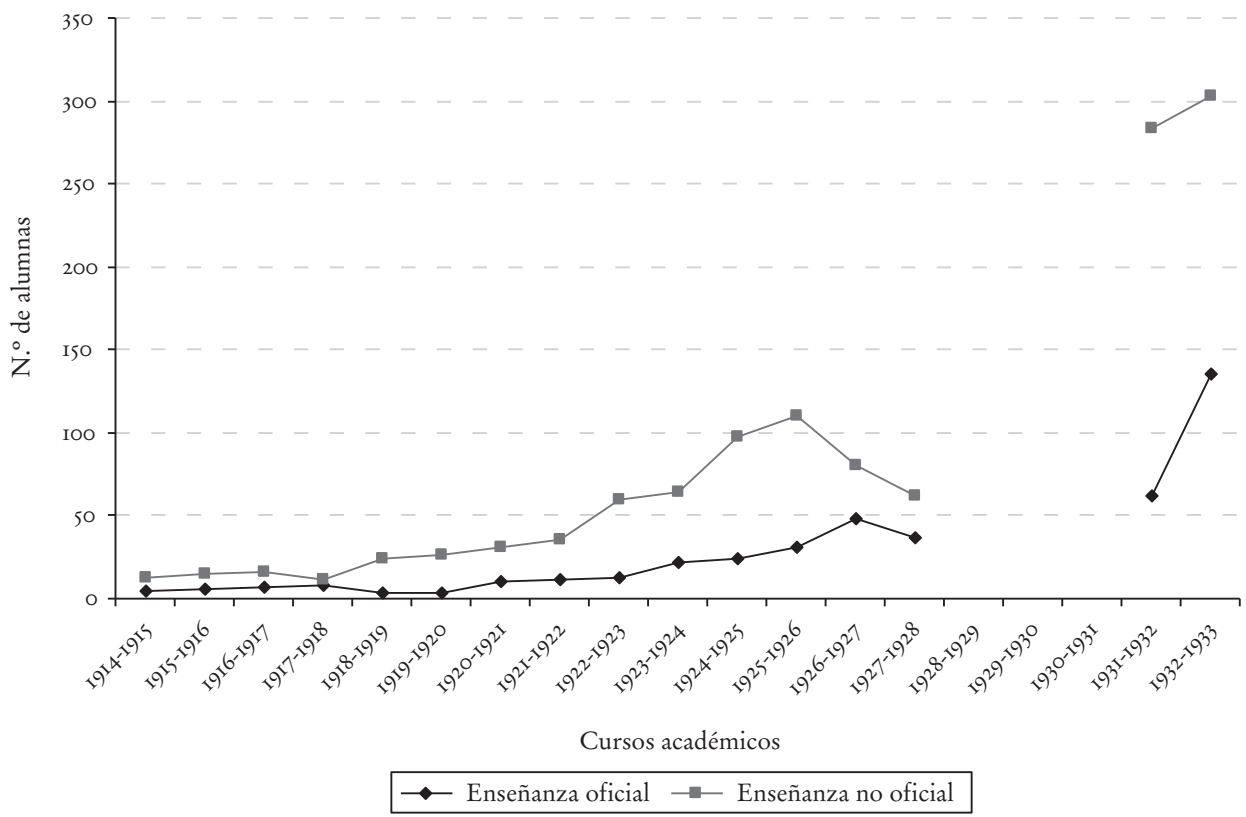

Fuente: Elaboración propia a partir de los datos del Ine. Anuarios Estadísticos de igis a 1934.

(C) EDICIONES UNIVERSIDAD DE SALAMANCA

Hist. educ., 36, 2017, pp. 277-300 
La creciente atención a los estudios de segunda enseñanza por parte de los diferentes gobiernos se ve reflejada en los datos que mostramos. Estos estudios estuvieron sometidos a continuos cambios que pretendían, con mayor o menor éxito, modernizarlos y adaptarlos a las circunstancias sociales del momento. Estos cambios afectaron a la mujer, pues las políticas del momento comenzaron a contemplarla, poco a poco, no sólo como ama de hogar, sino como miembro activo de la sociedad.

En las primeras décadas del siglo xx, la incorporación a la enseñanza secundaria de la mujer pacense fue tímida, pero paulatinamente comenzaron a tener más protagonismo en una época en la que todavía no se consideraba su formación en estos estudios de mucho valor. Al igual que pasaba en la provincia de Cáceres es en los primeros años de la Dictadura de Primo de Rivera cuando se observa un pico en el aumento de alumnas, y no fue hasta I931, con la II República, cuando se aprecia de nuevo un aumento en el número de alumnas matriculadas. Aun así, las alumnas que concurrían a las aulas del Instituto eran una minoría, pues, a pesar de que la normativa las admitía, hubo poca demanda femenina en este nivel educativo y cierta reticencia hacia su asistencia a los institutos acompañada por una preferencia por los colegios privados.

\subsection{Premios y menciones}

El acceso a la segunda enseñanza, como hemos visto, fue lento, pero se consiguió. El fruto del trabajo que realizaron estas primeras mujeres se ve reflejado en los premios y menciones que muchas consiguieron obtener por sus buenos resultados académicos. Cabe mencionar que no sólo se premiaba el esfuerzo a las alumnas de la enseñanza oficial, sino que también se contemplaban los resultados obtenidos en la enseñanza no oficial; pues se trataba de un reconocimiento hacia la labor desarrollada por el alumnado independientemente de la modalidad por la que hubieran optado para cursar los estudios, como así se ha podido constatar a través de las memorias de los centros que se leían en los actos de inauguración del nuevo curso escolar.

Los premios se concebían como estímulo para el alumnado más sobresaliente, se comunicaban públicamente y, normalmente, se les concedían a aquellos que habían obtenido la calificación de sobresaliente. Estos premios podían obtenerse desde un primer momento, es decir, porque se había obtenido la calificación de sobresaliente en la asignatura o podían optar a la Matrícula de Honor sometiéndose a un nuevo examen.

A continuación, mostramos unas tablas con las alumnas que obtuvieron recompensa por sus estudios y las asignaturas en las que destacaron, así como de qué tipo de enseñanza provenían. 
LA INCORPORACIÓN DE LAS MUJERES A LA SEGUNDA ENSEÑANZA PÚBLICA

EN EXTREMADURA (I900-I936)

CRISTINA REDONDO CASTRO, ISABEL GRANA GIL Y CARMEN SANCHIDRIÁN BLANCO

Tabla Iv: Premios y menciones a las alumnas del Instituto de Cáceres

\begin{tabular}{|c|c|c|}
\hline ALUMNAS & ASIGNATURAS & ENSEÑANZA \\
\hline \multicolumn{3}{|c|}{ CuRso I9I6-I7 } \\
\hline Felisa Izquierdo Macayo & I. ${ }^{\circ}$ Religión & \\
\hline Juana Izquierdo Macayo & I. ${ }^{\circ}$ Religión & \\
\hline Emilia Anaya Fuentes & Geografía especial de España & \\
\hline Juana Rosado Álvarez de Sotomayor & $\begin{array}{l}\text { Elementos de H. }{ }^{a} \text { General de la Literatura } \\
\text { 2. }{ }^{\circ} \text { Dibujo }\end{array}$ & \\
\hline \multicolumn{3}{|c|}{ CuRSO I925-26 } \\
\hline Ángela Blázquez Izquierdo & $\begin{array}{l}\text { Lengua Castellana } \\
\text { Nociones de Aritmética y Geometría } \\
\text { I. }{ }^{\text {R Religión }} \\
\end{array}$ & OFICIAL \\
\hline Antonia Luengo y Luengo & $\begin{array}{l}\text { I. }{ }^{\circ} \text { Religión } \\
\text { Caligrafía }\end{array}$ & OFICIAL \\
\hline Társila Leal Osuna & 2. ${ }^{\circ}$ Religión & OFICIAL \\
\hline Narcisa García Tomé & 2. ${ }^{\circ}$ Religión & OFICIAL \\
\hline Florencia Rodríguez Rebollo & $\begin{array}{l}\text { 2. }{ }^{\circ} \text { Latín } \\
\text { Historia de España } \\
\text { I. }{ }^{\circ} \text { Francés } \\
3 .{ }^{\circ} \text { Religión } \\
\end{array}$ & OFICIAL \\
\hline Basilia Caldera Llopis & $\begin{array}{l}\text { 2. }{ }^{\circ} \text { Latín } \\
\text { Historia de España } \\
\text { I. }{ }^{\circ} \text { Francés } \\
3^{\circ} \text { Religión } \\
\end{array}$ & OFICIAL \\
\hline María del Carmen Revilla Montero & $\begin{array}{l}\text { I. }^{\circ} \text { Francés } \\
\text { Historia de España } \\
\text { 2. }{ }^{\circ} \text { Latín }\end{array}$ & NO OFICIAL \\
\hline Tomasa Andrada Zabas & $\begin{array}{l}\text { Receptiva y Composición } \\
\text { Fisiología e Higiene } \\
\text { Psicología y Lógica } \\
\end{array}$ & NO OFICIAL \\
\hline Cándida Prado González & I. ${ }^{\circ}$ Latín & NO OFICIAL \\
\hline María Gutiérrez del Egido & Psicología y Lógica & NO OFICIAL \\
\hline Luisa Jurado Carro & Física & NO OFICIAL \\
\hline María Rodríguez Carreño & 2. ${ }^{\circ}$ Francés & NO OFICIAL \\
\hline \multicolumn{3}{|c|}{ CuRso 1933-34 } \\
\hline Francisca Adames Belvís & 2. ${ }^{\circ}$ Curso-Plan de 1932 & OFICIAL \\
\hline María Gil Julián & $\begin{array}{l}\text { I. }{ }^{\circ} \text { Lengua Española } \\
\text { I. }{ }^{\circ} \text { Matemáticas } \\
\text { I. }{ }^{\circ} \text { Ciencias Naturales } \\
\end{array}$ & OFICIAL \\
\hline Inés Durán Fernández & I. ${ }^{\circ}$ Lengua Española & OFICIAL \\
\hline Rita Mayordomo Avilés & I. ${ }^{\circ}$ Lengua Española & OFICIAL \\
\hline Ligia López Sánchez & I. ${ }^{\circ}$ Ciencias Naturales & OFICIAL \\
\hline
\end{tabular}


LA INCORPORACIÓN DE LAS MUJERES A LA SEGUNDA ENSEÑANZA PÚBLICA

EN EXTREMADURA (I900-I936)

CRISTINA REDONDO CASTRO, ISABEL GRANA GIL Y CARMEN SANCHIDRIÁN BLANCO

\begin{tabular}{|c|c|c|}
\hline ALUMNAS & ASIGNATURAS & ENSEÑANZA \\
\hline Marcela Montero Moreno & 2. ${ }^{\circ}$ Lengua Española & \begin{tabular}{|l|} 
OFICIAL \\
\end{tabular} \\
\hline Valentina Ovejero Morales & $\begin{array}{l}\text { 2. }{ }^{\circ} \text { Lengua Española } \\
\text { 2. }{ }^{\circ} \text { Ciencias Naturales }\end{array}$ & OFICIAL \\
\hline M. a del Carmen Sánchez López & $\begin{array}{l}\text { 2. }{ }^{\circ} \text { Latín } \\
\text { I. }{ }^{\circ} \text { Francés } \\
\text { Geometría }\end{array}$ & OFICIAL \\
\hline Celia García Tomé & $\begin{array}{l}\text { 2. }{ }^{\circ} \text { Latín } \\
\text { Historia de España } \\
\text { Geometría }\end{array}$ & OFICIAL \\
\hline Rosa Montero Lucas & $\begin{array}{l}\text { 2. }{ }^{\circ} \text { Latín } \\
\text { Geometría }\end{array}$ & OFICIAL \\
\hline Marcelina Martín Mateos & $\begin{array}{l}\text { 2. }{ }^{\circ} \text { Latín } \\
\text { I. }{ }^{\circ} \text { Francés } \\
\text { Geometría }\end{array}$ & OFICIAL \\
\hline Luisa Alonso López & $\begin{array}{l}2^{\circ}{ }^{\circ} \text { Latín } \\
\text { Historia Universal }\end{array}$ & OFICIAL \\
\hline Adela Macías Hurtado & I. ${ }^{\circ}$ Francés & OFICIAL \\
\hline Emeteria Pavón Vicho & Geometría & OFICIAL \\
\hline M. ${ }^{a}$ del Carmen Yagüe Peñalva & 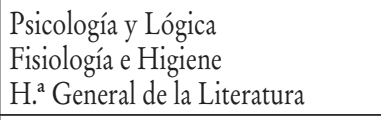 & OFICIAL \\
\hline Josefa Ortega Pereda-Velasco & H. ${ }^{a}$ General de la Literatura & OFICIAL \\
\hline M. del Pilar Blázquez Román & $\begin{array}{l}2 .^{\circ} \text { Latín } \\
\text { Perceptiva y Composición }\end{array}$ & NO OFICIAL \\
\hline Ramona Nebrada Martín & $\begin{array}{l}\text { I. }{ }^{\circ} \text { Francés } \\
\text { I. }{ }^{\circ} \text { Latín } \\
\text { 2. } .^{\circ} \text { Latín } \\
\text { Perceptiva y Composición } \\
\end{array}$ & NO OFICIAL \\
\hline María Beatriz Rubio Bermejo & Geometría & NO OFICIAL \\
\hline Carmen Mardones Melara & Ingreso & \\
\hline \multicolumn{3}{|c|}{ CURSO I934-35 } \\
\hline M. a del Carmen Gil de la Larrázabal & Ingreso & \\
\hline Concepción de Vega y Ruiz & Primer curso-Plan 1932 & OFICIAL \\
\hline Soledad Jacinto Muñoz & Primer curso-Plan 1932 & OFICIAL \\
\hline María Gil Julián & Segundo curso-Plan I932 & OFICIAL \\
\hline Ignacia Leno Granado & Tercer curso-Plan I932 & OFICIAL \\
\hline Francisca Adame Belvis & Tercer curso-Plan 1932 & OFICIAL \\
\hline M. a del Carmen Sánchez López & $\begin{array}{l}\text { Perceptiva y Composición } \\
2^{\circ}{ }^{\circ} \text { Francés } \\
\text { Álgebra y Trigonometría }\end{array}$ & OFICIAL \\
\hline Celia García Tomé & $\begin{array}{l}\text { Perceptiva y Composición } \\
\text { Historia Universal }\end{array}$ & OFICIAL \\
\hline
\end{tabular}


LA INCORPORACIÓN DE LAS MUJERES A LA SEGUNDA ENSEÑANZA PÚBLICA

EN EXTREMADURA (I900-I936)

CRISTINA REDONDO CASTRO, ISABEL GRANA GIL Y CARMEN SANCHIDRIÁN BLANCO

\begin{tabular}{|c|c|c|}
\hline ALUMNAS & ASIGNATURAS & ENSEÑANZA \\
\hline Carolina Muñoz Díaz & Perceptiva y Composición & OFICIAL \\
\hline Marcelina Martín Mateos & I. ${ }^{\circ}$ Religión & OFICIAL \\
\hline Josefa Marín Collar & I. ${ }^{\circ}$ Religión & OFICIAL \\
\hline Emeteria Pavónvicho & I. ${ }^{\circ}$ Religión & OFICIAL \\
\hline María de la Piedad Núñez Ortiz & $\begin{array}{l}\text { H. a General de la Literatura } \\
\text { Física } \\
\text { Fisiología e Higiene }\end{array}$ & OFICIAL \\
\hline Luisa Alonso López & \begin{tabular}{|l|} 
Física \\
Fisiología e Higiene
\end{tabular} & OFICIAL \\
\hline Vicente Gonzalo Ojalvo & Química General & OFICIAL \\
\hline María Lourdes Rodríguez Carrasco & Primer curso-Plan 1932 & NO OFICIAL \\
\hline Carmen Mandome Delgado & Historia Universal & NO OFICIAL \\
\hline Ramona Nebrada Martín & $\begin{array}{l}\text { H. }{ }^{a} \text { General de la Literatura } \\
\text { Física }\end{array}$ & NO OFICIAL \\
\hline Carmen Marqués Tejano & Física & NO OFICIAL \\
\hline M. del Pilar Blázquez Rodríguez & H. ${ }^{a}$ General de la Literatura & NO OFICIAL \\
\hline Pura Bautista Amador & Física & NO OFICIAL \\
\hline \multicolumn{3}{|c|}{ CURSO $1935-36$} \\
\hline Manuela Criado Muriel & Beca 3. ${ }^{\circ}$ Curso & \\
\hline Isabel Arias Mateo & Beca $4 .{ }^{\circ}$ Curso & \\
\hline Joaquina Civantos Sabatier & Beca 4. ${ }^{\circ}$ Curso & \\
\hline M. a del Carmen Gil de Larrazabal & Primer curso-Plan 1932 & OFICIAL \\
\hline Josefa Crespo Román & Segundo curso-Plan 1932 & OFICIAL \\
\hline Soledad Jacinto Muñoz & Segundo curso-Plan 1932 & OFICIAL \\
\hline María Gil Julián & Tercer curso-Plan 1932 & OFICIAL \\
\hline Francisca Adame Belvis & Cuarto curso-Plan 1932 & OFICIAL \\
\hline Celia García Tomé & Psicología y Lógica & OFICIAL \\
\hline M. del Carmen Sánchez López & $\begin{array}{l}\text { H. a General de la Literatura } \\
\text { Física }\end{array}$ & OFICIAL \\
\hline Marcelina Martín Mateos & Física & OFICIAL \\
\hline Araceli Cuesta Delgado & Fisiología e Higiene & OFICIAL \\
\hline M. ${ }^{a}$ de la Piedad Núñez Ortiz & $\begin{array}{l}\text { Ética y Rudimentos del Derecho } \\
\text { Historia Natural } \\
\text { Agricultura y Técnica } \\
\text { Química General }\end{array}$ & OFICIAL \\
\hline Luisa Alonso López & $\begin{array}{l}\text { Ética y Rudimentos del Derecho } \\
\text { Química General } \\
\end{array}$ & OFICIAL \\
\hline Ana González Solís & Química General & NO OFICIAL \\
\hline
\end{tabular}

Fuente: Elaboración propia a partir de los datos obtenidos de las Memorias Escolares del Instituto de Cáceres de los cursos que se expresan. 
LA INCORPORACIÓN DE LAS MUJERES A LA SEGUNDA ENSEÑANZA PÚBLICA

EN EXTREMADURA (I900-I936)

CRISTINA REDONDO CASTRO, ISABEL GRANA GIL Y CARMEN SANCHIDRIÁN BLANCO

Tabla v: Premios y menciones a las alumnas del Instituto de Badajoz

\begin{tabular}{|c|c|c|}
\hline ALUMNAS & ASIGNATURAS & ENSEÑANZA \\
\hline \multicolumn{3}{|c|}{ CURSO I9II-I2 } \\
\hline Clotilde Márquez Cubero & 2. ${ }^{\circ}$ Religión & NO OFICIAL \\
\hline Enriqueta Martín Ortiz & $\begin{array}{l}{ }^{\circ}{ }^{\circ} \text { Latín } \\
\text { I. }{ }^{\circ} \text { Francés }\end{array}$ & NO OFICIAL \\
\hline \multicolumn{3}{|c|}{ CURSO I9I2-I3 } \\
\hline M. a de la Soledad Martínez & Ingreso & \\
\hline Obdulia Fernández & Ingreso & \\
\hline María de las Mercedes Ruiz & $\begin{array}{l}\text { Castellano } \\
\text { Nociones de Aritmética y Geometría } \\
\text { I. }{ }^{\circ} \text { Religión }\end{array}$ & NO OFICIAL \\
\hline María F. Grandizo Martín & Geografía general y de Europa & NO OFICIAL \\
\hline Ángela Cabanillas Daza & I. ${ }^{\circ}$ Francés & NO OFICIAL \\
\hline Enriqueta Martín Ortiz & $\begin{array}{l}\text { Preceptiva y Composición } \\
\text { 2. } .^{\circ} \text { Francés } \\
\text { Historia Universal }\end{array}$ & NO OFICIAL \\
\hline Clotilde Márquez Cubero & $3 .^{\circ}$ Religión & NO OFICIAL \\
\hline \multicolumn{3}{|c|}{ CURSO I9I3-I4 } \\
\hline Obdulia Fernández de la Cerda & $\begin{array}{l}\text { Castellano } \\
\text { Geografía general y de Europa } \\
\text { Nociones de Aritmética y Geometría } \\
\text { I. }{ }^{\circ} \text { Religión } \\
\end{array}$ & OFICIAL \\
\hline Magdalena Fernández de la Cerda & $\begin{array}{l}\text { Castellano } \\
\text { Geografía general y de Europa } \\
\text { Nociones de Aritmética y Geometría } \\
\text { I. }{ }^{\circ} \text { Religión } \\
\end{array}$ & OFICIAL \\
\hline M. ${ }^{a}$ Soledad Martínez y Martínez & $\begin{array}{l}\text { Castellano } \\
\text { I. }^{\circ} \text { Religión } \\
\text { Nociones de Aritmética y Geometría } \\
\text { Caligrafía } \\
\end{array}$ & NO OFICIAL \\
\hline María de las Mercedes Ruiz & I. ${ }^{\circ}$ Latín & NO OFICIAL \\
\hline Antonia Arqueros Galán & Historia de España & OFICIAL \\
\hline \multicolumn{3}{|c|}{ CURSO I9I4-IS } \\
\hline Nicolasa López Arroyo & \begin{tabular}{|l|} 
Castellano \\
Geografía general y de Europa \\
Nociones de Aritmética y Geometría \\
I. ${ }^{\circ}$ Religión \\
Caligrafía \\
\end{tabular} & OFICIAL \\
\hline Dolores Carrasco Pérez & $\begin{array}{l}\text { Geografía general y de Europa } \\
\text { Caligrafía }\end{array}$ & COLEGIADA \\
\hline Enriqueta Martín Ortiz & Historia Natural & LIBRE \\
\hline Carmen Peralta Villar & $\begin{array}{l}\text { I. }^{\circ} \text { Religión } \\
\text { Historia Universal } \\
\text { Preceptiva } \\
\text { Álgebra y Trigonometría }\end{array}$ & OFICIAL \\
\hline
\end{tabular}


LA INCORPORACIÓN DE LAS MUJERES A LA SEGUNDA ENSEÑANZA PÚBLICA

EN EXTREMADURA (I900-I936)

CRISTINA REDONDO CASTRO, ISABEL GRANA GIL Y CARMEN SANCHIDRIÁN BLANCO

\begin{tabular}{|c|c|c|}
\hline ALUMNAS & ASIGNATURAS & ENSEÑANZA \\
\hline Obdulia Fernández de la Cerda & $\begin{array}{l}\text { 2. }{ }^{\circ} \text { Latín } \\
\text { 2. }{ }^{\circ} \text { Religión } \\
\text { Geografía especial de España } \\
\text { Aritmética }\end{array}$ & OFICIAL \\
\hline Magdalena Fernández de la Cerda & $\begin{array}{l}{ }^{\circ}{ }^{\circ} \text { Latín } \\
2 .{ }^{\circ} \text { Religión } \\
\text { Geografía especial de España } \\
\text { Aritmética }\end{array}$ & OFICIAL \\
\hline M. Soledad Martínez & $\begin{array}{l}\text { I. }{ }^{\circ} \text { Latín } \\
\text { 2. }{ }^{\circ} \text { Religión } \\
\text { Geografía especial de España }\end{array}$ & LIBRE \\
\hline María de las Mercedes Ruiz & 3. ${ }^{\circ}$ Religión & COLEGIADA \\
\hline Antonia Arqueros Galán & $\begin{array}{l}\text { Historia Universal } \\
\text { I. }{ }^{\circ} \text { Dibujo }\end{array}$ & OFICIAL \\
\hline \multicolumn{3}{|c|}{ CuRso I9I6-I7 } \\
\hline Fermina Velarde Hidalgo & $\begin{array}{l}\text { Castellano } \\
\text { Geografía general y de Europa } \\
\text { Nociones de Aritmética y Geometría } \\
\text { I. Religión } \\
\text { Caligrafía } \\
\end{array}$ & OFICIAL \\
\hline Teresa Rebollo Amaro & $\begin{array}{l}\text { Castellano } \\
\text { I. }{ }^{\text {o Religión }} \\
\text { Geografía general y de Europa } \\
\text { Historia de España } \\
\text { Nociones de Aritmética y Geometría }\end{array}$ & NO OFICIAL \\
\hline María Cerrato Rodríguez & $\begin{array}{l}\text { Geografía general y de Europa } \\
\text { Geografía especial de España } \\
\text { Historia de España }\end{array}$ & LIBRE \\
\hline María Cruz Navarro & Nociones de Aritmética y Geometría & OFICIAL \\
\hline M. a del Carmen Ruiz & Aritmética & NO OFICIAL \\
\hline Nicolasa López Arroyo & $\begin{array}{l}\text { 2. }{ }^{\circ} \text { Latín } \\
\text { I. }{ }^{\circ} \text { Francés } \\
\text { Historia de España } \\
\text { Geometría } \\
3 .{ }^{\circ} \text { Religión }\end{array}$ & OFICIAL \\
\hline Dolores Carrasco Pérez & I. ${ }^{\circ}$ Francés & NO OFICIAL \\
\hline Obdulia Fernández de la Cerda & $\begin{array}{l}\text { Preceptiva } \\
\text { 2. }{ }^{\circ} \text { Francés } \\
\text { I. }^{\circ} \text { Dibujo } \\
\text { Historia Universal } \\
\text { Álgebra y Trigonometría } \\
\end{array}$ & OFICIAL \\
\hline Magdalena Fernández de la Cerda & $\begin{array}{l}\text { Preceptiva } \\
\text { I. Dibujo } \\
\text { Álgebra y Trigonometría } \\
\text { 2. }{ }^{\circ} \text { Francés } \\
\end{array}$ & OFICIAL \\
\hline
\end{tabular}


LA INCORPORACIÓN DE LAS MUJERES A LA SEGUNDA ENSEÑANZA PÚBLICA

EN EXTREMADURA (I900-I936)

CRISTINA REDONDO CASTRO, ISABEL GRANA GIL Y CARMEN SANCHIDRIÁN BLANCO

\begin{tabular}{|c|c|c|}
\hline ALUMNAS & ASIGNATURAS & \begin{tabular}{|l} 
ENSEÑANZA \\
\end{tabular} \\
\hline M. Soledad Martínez & $\begin{array}{l}\text { Preceptiva } \\
2{ }^{\circ} \text { Francés } \\
\text { I. }{ }^{\circ} \text { Dibujo } \\
\text { Historia Universal } \\
\text { Álgebra y Trigonometría }\end{array}$ & LIBRE \\
\hline Carmen Peralta Villar & $\begin{array}{l}\text { Historia Natural } \\
\text { Agricultura }\end{array}$ & OFICIAL \\
\hline \multicolumn{3}{|c|}{ CuRso I919-20 } \\
\hline Elena Felipe González & $\begin{array}{l}\text { Castellano } \\
\text { Geografía general y de Europa } \\
\text { Geografía especial de España }\end{array}$ & NO OFICIAL \\
\hline Sinforosa Antúnez Bautista & $\begin{array}{l}\text { Geografía general y de Europa } \\
\text { Geografía especial de España }\end{array}$ & NO OFICIAL \\
\hline Amalia Blanco Carrasco & $\begin{array}{l}\text { Geografía especial de España } \\
\text { Álgebra y Trigonometría }\end{array}$ & NO OFICIAL \\
\hline Aurelia Higuera Martín & Preceptiva y Composición & NO OFICIAL \\
\hline Fermina Velarde Hidalgo & $\begin{array}{l}\text { 2. }{ }^{\circ} \text { Francés } \\
\text { Historia Universal } \\
\text { Álgebra y Trigonometría } \\
\text { I. }{ }^{\circ} \text { Dibujo } \\
\end{array}$ & OFICIAL \\
\hline María Cruz Navarro & $\begin{array}{l}\text { Historia Literaria } \\
\text { Física } \\
\text { Fisiología e Higiene } \\
2{ }^{\circ} \text { Dibujo } \\
\end{array}$ & OFICIAL \\
\hline \multicolumn{3}{|c|}{ CURSO I920-2I } \\
\hline Dolores Hidalgo Gómez & $\begin{array}{l}\text { Castellano } \\
\text { I. }{ }^{\circ} \text { Religión }\end{array}$ & OFICIAL \\
\hline Rafaela Martín Ruiz & Castellano & OFICIAL \\
\hline Socorro Blanco Carrasco & Castellano & OFICIAL \\
\hline Adela Navarro Patiño & \begin{tabular}{|l|} 
Castellano \\
I. $^{\text {o Religión }}$ \\
Geografía especial y de Europa \\
\end{tabular} & NO OFICIAL \\
\hline María Bourrelier Fernández & \begin{tabular}{|l|} 
Castellano \\
I. $^{\text {o Religión }}$ \\
Geografía especial y de Europa \\
\end{tabular} & OFICIAL \\
\hline Griselda Velarde Hidalgo & $\begin{array}{l}\text { Geografía general de Europa } \\
\text { I. }{ }^{\circ} \text { Religión } \\
\end{array}$ & OFICIAL \\
\hline Antonia Checa Linares & Aritmética & NO OFICIAL \\
\hline Elena Felipe González & \begin{tabular}{|l|} 
I. $^{o}$ Francés \\
Historia de España \\
Geometría \\
\end{tabular} & NO OFICIAL \\
\hline Amalia Martín González & Historia de España & OFICIAL \\
\hline María Henso Haba & Historia de España & NO OFICIAL \\
\hline
\end{tabular}


LA INCORPORACIÓN DE LAS MUJERES A LA SEGUNDA ENSEÑANZA PÚBLICA

EN EXTREMADURA (I900-I936)

CRISTINA REDONDO CASTRO, ISABEL GRANA GIL Y CARMEN SANCHIDRIÁN BLANCO

\begin{tabular}{|c|c|c|}
\hline ALUMNAS & ASIGNATURAS & ENSEÑANZA \\
\hline Fermina Velarde Hidalgo & $\begin{array}{l}\text { Fisiología e Higiene } \\
\text { Física } \\
\text { 2. }{ }^{\circ} \text { Dibujo } \\
\end{array}$ & OFICIAL \\
\hline Amalia Blanco Carrasco & Historia Literaria & OFICIAL \\
\hline \multicolumn{3}{|c|}{ CURSO I92I-22 } \\
\hline M. a Josefa Crisóstomo González & I. Religión & NO OFICIAL \\
\hline Rafaela Martín Ruiz & I. ${ }^{\circ}$ Latín & OFICIAL \\
\hline María Bourrelier Fernández & $\begin{array}{l}\text { I. Latín } \\
\text { Geografía especial de España }\end{array}$ & OFICIAL \\
\hline María Ignacia González & I. ${ }^{\circ}$ Latín & NO OFICIAL \\
\hline Griselda Velarde Hidalgo & Geografía especial de España & OFICIAL \\
\hline Eugenia Alba Carballo & Geografía especial de España & \begin{tabular}{|l|} 
OFICIAL \\
\end{tabular} \\
\hline Adela Navarro Patiño & Preceptiva y Composición & NO OFICIAL \\
\hline Sinforosa Antúnez Bautista & 2. ${ }^{\circ}$ Francés & NO OFICIAL \\
\hline Elena Felipe González & $\begin{array}{l}\text { Historia Universal } \\
\text { Historia Literaria } \\
\end{array}$ & NO OFICIAL \\
\hline Fermina Velarde Hidalgo & $\begin{array}{l}\text { Ética y Rudimento de Derecho } \\
\text { Historia Natural } \\
\text { Agricultura } \\
\text { Química }\end{array}$ & OFICIAL \\
\hline
\end{tabular}

Fuente: Elaboración propia a partir de los datos de las Memorias Escolares del Instituto de Badajoz de los cursos que se expresan.

Como podemos observar, una mayoría de las alumnas premiadas habían realizado sus estudios en el propio Instituto. Tomando como ejemplo el curso I925-26, del Instituto de Cáceres, de los 26 premios otorgados un 61,5\% correspondían a la enseñanza oficial y un $38,5 \%$ a la no oficial, siendo siempre la cifra más elevada en la primera. En el caso de Badajoz, nos encontramos con la misma situación, además, si comparamos los premios conseguidos por las alumnas inscritas con los datos de la Tabla iv, podemos ver cómo la enseñanza oficial no sólo conseguía un mayor número de alumnas premiadas, sino que casi un 90\% de ellas lo conseguían: por ejemplo, en el curso escolar de I9I4-is las 5 alumnas matriculadas en la enseñanza oficial consiguieron reconocimiento en alguna asignatura. Estos datos dejan entrever que las alumnas que accedían a la segunda enseñanza lo hacían con vistas a ir más allá de estos estudios. Asimismo, podemos apreciar que en su mayoría los premios eran concedidos en asignaturas de letras, mientras que las de carácter científico y técnico eran las menores. Podemos destacar el caso de Fermina Velarde Velasco, alumna del Instituto de Badajoz, una alumna sobresaliente que, a pesar de no tener información de todos los cursos académicos, podemos ver cómo fue reconocido su esfuerzo, terminó el bachillerato y cursó posteriormente la licenciatura de Ciencias Naturales, llegando a ser profesora del mismo centro donde cursó sus estudios. 


\section{Reflexiones finales}

Con este trabajo se ha intentado ofrecer una aproximación acerca de la incorporación de la mujer a los estudios secundarios de los Institutos Históricos extremeños, concretamente en los de Cáceres y Badajoz, en el primer tercio del siglo xx. Principalmente, el foco de interés se ha centrado en conocer la evolución que experimentó esa incorporación de las mujeres a la educación secundaria y las causas que favorecieron o dificultaron su desarrollo.

En Extremadura, esa incorporación fue más lenta y menos decidida que en otras regiones debido sobre todo a las circunstancias de la región en la que las tasas de analfabetismo eran muy altas en general y realmente preocupantes las de las mujeres. En este sentido, hay que entender que el esfuerzo mayor fuera hacia la alfabetización y escolarización de la población en edad escolar y las clases de adultos. Además, la población era fundamentalmente rural y dispersa y los institutos estaban en las capitales, por lo que una parte importante de la población no tenía opción de acceder a este tipo de enseñanza y mucho menos si era mujer.

A través de los datos recopilados por los Anuarios Estadísticos y las Memorias, hemos podido apreciar como en la etapa la incorporación a los estudios secundarios se disparó y la enseñanza oficial se vio favorecida.

No obstante, podríamos afirmar que, desde el inicio del nuevo siglo, las mujeres, por primera vez, pudieron acceder a la segunda enseñanza, como recoge Herrero, «no como excepción ni cómo anécdota, sino como estudiantes de pleno derecho, al mismo nivel que sus compañeros varones ${ }^{27}$. Este hecho marcó un antes y un después en la educación de las mujeres, ya que el poder acceder a todos los grados de la enseñanza en las mismas condiciones que los niños potenció la igualdad educativa y, con ella, las mujeres vieron que tenían una nueva posibilidad de acceso a la instrucción, a la vida pública y al mundo profesional. Es preciso continuar este estudio incluyendo el acceso de las mujeres tanto a los nuevos institutos elementales que se crearon en estos años en estas provincias como a la enseñanza secundaria no oficial, lo que nos permitirá tener un mejor conocimiento de este tema.

27 Herrero SÁenz, R.: «La incorporación de las mujeres a la educación secundaria durante la Segunda República: Un estudio de casos sobre el Instituto Quevedo de Madrid», en LóPEz-Ocón, L.: Aulas modernas. Nuevas perspectivas sobre las reformas de la enseñanza secundaria en la época de la JAE (1907-1939), Madrid, Editorial Dykinson, 20I4, pp. 246-247. 